\title{
Biosynthesis and engineering of kaempferol in Saccharomyces cerevisiae
}

\author{
Lijin Duan ${ }^{1,2+}$, Wentao Ding ${ }^{2+}$, Xiaonan Liu ${ }^{2,3}$, Xiaozhi Cheng ${ }^{2}$, Jing Cai ${ }^{4}$, Erbing Hua ${ }^{1 *}$ and Huifeng Jiang ${ }^{2^{*}}$
}

\begin{abstract}
Background: Kaempferol is a flavonol with broad bioactivity of anti-oxidant, anti-cancer, anti-diabetic, anti-microbial, cardio-protective and anti-asthma. Microbial synthesis of kaempferol is a promising strategy because of the low content in primary plant source.

Methods: In this study, the biosynthesis pathway of kaempferol was constructed in the budding yeast Saccharomyces cerevisiae to produce kaempferol de novo, and several biological measures were taken for high production.

Results: Firstly, a high efficient flavonol synthases (FLS) from Populus deltoides was introduced into the biosynthetic pathway of kaempferol. Secondly, a S. cerevisiae recombinant was constructed for de novo synthesis of kaempferol, which generated about $6.97 \mathrm{mg} / \mathrm{L}$ kaempferol from glucose. To further promote kaempferol production, the acetyl-CoA biosynthetic pathway was overexpressed and p-coumarate was supplied as substrate, which improved kaempferol titer by about 23 and 120\%, respectively. Finally, a fed-batch process was developed for better kaempferol fermentation performance, and the production reached $66.29 \mathrm{mg} / \mathrm{L}$ in $40 \mathrm{~h}$.
\end{abstract}

Conclusions: The titer of kaempferol in our engineered yeast is 2.5 times of the highest reported titer. Our study provides a possible strategy to produce kaempferol using microbial cell factory.

Keywords: Kaempferol, Flavonol synthase, Acetyl-CoA, Saccharomyces cerevisiae

\section{Background}

Kaempferol is a polyphenol anti-oxidant found in many edible plants, which have been commonly used in traditional medicine (e.g. Ginkgo biloba, Tilia spp., Equisetum spp., Moringa oleifera, Sophora japonica and propolis) [1, 2]. Dietary kaempferol has attracted extensive attention because of the beneficial effects on human health, including anti-oxidant, anti-inflammatory, anti-microbial, anticancer, cardio-protective, neuro-protective, anti-diabetic, anti-osteoporotic, estrogenic, anti-estrogenic, anxiolytic, analgesic and anti-allergic activities [1-4]. Interestingly, although kaempferol inhibits cancer cell growth and induces cancer cell apoptosis, it appears to preserve or

\footnotetext{
*Correspondence: huarb@tust.edu.cn; jiang_hf@tib.cas.cn ${ }^{\dagger}$ Lijin Duan and Wentao Ding contributed equally to this work

${ }^{1}$ Key Laboratory of Industrial Microbiology, College of Biotechnology, Tianjin University of Science \& Technology, Tianjin, China

${ }^{2}$ Key Laboratory of Systems Microbial Biotechnology, Tianjin Institute of Industrial Biotechnology, Chinese Academy of Sciences, Tianjin, China Full list of author information is available at the end of the article
}

protect normal cell viability [2]. However, the content of kaempferol in plants is very low, which results in high cost for kaempferol production from traditional plant extraction $[5,6]$. Thus, it is a promising alternative strategy to produce kaempferol using microbial cell factory.

Biosynthetic pathway of kaempferol has been identified in plant [5]. At the initial stage, phenylalanine is converted into $p$-coumaryl-CoA by phenylalanine ammonia lyase (PAL), cinnamic acid 4-hydroxylase $(\mathrm{C} 4 \mathrm{H})$ and 4-coumaric acid ligase (4CL). Then, naringenin is generated by condensation reaction between one molecular $p$-coumaryl-CoA and three molecules of malonyl-CoA by chalcone synthesis $(\mathrm{CHS})$ and chalcone isomerase $(\mathrm{CHI})$. At last, the naringenin is converted into kaempferol via dihydrokaempferol by flavanone $3 \beta$-hydroxylase (F3H) and flavonol synthase (FLS). Although kaempferol has been successfully synthesized by engineered microbes, the titer of kaempferol is still lower than many other microbial produced flavonoids, such as naringenin and eriodictyol [7-11]. One of the possible causes is that the 
carbon flux toward precursors is usually not enough [12]. Engineering metabolic pathway toward acetyl-CoA or malonyl-CoA would partially solve this problem [13-15]. In addition, the low efficiency of key enzymes in kaempferol biosynthetic pathway could also cause this issue. It has been reported that although the precursor naringenin was sufficiently supplied, the recombinant cell factory still produced kaempferol at a very low level [10].

In this study, we proposed to construct a yeast cell factory to improve kaempferol production. Firstly, we would screen higher efficient FLS from different plants to improve kaempferol conversion rate from the precursor naringenin. Secondly, the selected FLS would be combined with other pathway genes to build a microbial cell factory for de novo kaempferol synthesis. Moreover the acetyl-CoA and malonyl-CoA pathways would be engineered to further improve kaempferol biosynthesis. $p$-Coumarate would be also supplemented as substrate to improve the precursors supply. Finally, the fermentation condition would be optimized for better kaempferol production.

\section{Results and discussion}

\section{Biosynthesis of kaempferol from naringenin}

Despite the significant pharmaceutical effect of kaempferol, the low content in plant or engineered microbes has restricted its application. We firstly attempted to construct the kaempferol pathway in S. cerevisiae from naringenin, a common intermediate of flavonoid biosynthesis. Naringenin is converted into kaempferol via dihydrokaempferol, catalyzed with flavanone $3 \beta$-hydroxylase $(\mathrm{F} 3 \mathrm{H})$ and flavonol synthase (FLS) (Fig. 1a). In this research, F3H from Arabidopsis thaliana [16] and FLS from Malus domestica [17] (encoded by AtF3H and $M d F L S$ respectively) were expressed in S. cerevisiae to build a kaempferol-producing recombinant. Indeed, using (2S)-naringenin as substrate, the recombinant yeast expressing AtF3H produced (+)-dihydrokaempferol (Fig. 1b, c). On the other hand, both (+)-dihydrokaempferol and kaempferol were detected in the medium fermented by the recombinant expressing $A t F 3 H$ and MdFLS (Fig. 1b, d). Moreover, the molecular weights of (+)-dihydrokaempferol and kaempferol were confirmed by liquid chromatograph-mass spectrometer (LC-MS) (Fig. 1c, d). However, when $100 \mathrm{mg} / \mathrm{L}$ initial (2S)-naringenin was supplemented, $23.62 \mathrm{mg} / \mathrm{L}(+)$-dihydrokaempferol and only $6.03 \mathrm{mg} / \mathrm{L}$ kaempferol were produced through $24 \mathrm{~h}$ conversion. The relatively low titer of kaempferol indicated that the step from $(+)$-dihydrokaempferol to kaempferol is the bottleneck of kaempferol production from naringenin.

To solve this problem, we proposed to find a more efficient FLS from other plant sources. FLS genes from five plant species, including $A$. thaliana, Citrus unshiu, $M$. domestica, P. deltoides and Zea Mays [10, 18-21] (encoded by AtFLS, CitFLS, MdFLS, PdFLS and ZmFLS, respectively), were overexpressed with $A t F 3 H$ in yeast, respectively. The viability of these FLS in kaempferol production were compared according to the final kaempferol productions in whole cell catalysis. The strain expressing PdFLS resulted in the highest kaempferol production $(12.98 \mathrm{mg} / \mathrm{L}$, Fig. 1e) and the lowest dihydrokaempferol accumulation (16.77 mg/L, Fig. 1e), indicating that PdFLS has the highest efficiency to produce kaempferol from dihydrokaempferol (Additional file 1: Figure S1A, $\mathrm{S} 1 \mathrm{~B})$. Besides, the recombinants expressing AtFLS and CitFLS produced lower kaempferol than that expressing PdFLS, but significant higher than that expressing $M d F L S$ and ZmFLS (Fig. 1e). According to the previous studies, FLS from $P$. deltoides, $A$. thaliana and $C$. unshiu performed both FLS and F3H function, which are able to use naringenin as a substrate to produce kaempferol as well as dihydrokaempferol [20-23]. However, this F3H function was not observed in FLS from $M$. domestica and $Z$. Mays $[17,18]$. This could partially explain that the recombinants expressing PdFLS, AtFLS and CitFLS produced significant higher kaempferol than those expressing MdFLS and ZmFLS. Although the sole expression of bifunctional FLS would enable the recombinant to convert naringenin into kaempferol, the co-expression with F3H would promote flavonol titer and has been widely adopted in the previous studies [9, 24]. Considering the co-expression of PdFLS and AtF3H resulted in the highest kaempferol production from naringenin, this gene cluster was retained for further kaempferol biosynthesis.

\section{Construction of de novo synthetic pathway of kaempferol}

Based on the high efficient kaempferol conversion with AtF3H and PdFLS, we further attempted to build a pathway for de novo biosynthesis of kaempferol. A naringenin synthesis strain, W3NP, was constructed as parent strain through expressing phenylalanine ammonia lyase, cinnamic acid 4-hydroxylase, 4-coumaric acid ligase, chalcone synthase, and chalcone isomerase from Erigeron breviscapus (encoded by PAL, C4H, 4CL, CHS, CHI, respectively). Indeed, W3NP was able to synthesize naringenin from glucose, which was confirmed by LC-MS and ultraviolet (UV) absorption (Fig. 2b and Additional file 1: Figure S2). Thus, by introducing AtF3H and PdFLS to W3NP, a yeast recombinant W3NP-FF was generated, which carries a pathway for de novo kaempferol biosynthesis (Fig. 2c). In batch fermentation, W3NP produced $2.29 \mathrm{mg} / \mathrm{L}$ naringenin, while W3NP-FF produced $6.97 \mathrm{mg} / \mathrm{L}$ kaempferol, which was accompanied with $3.55 \mathrm{mg} / \mathrm{L}$ dihydrokaempferol and $3.32 \mathrm{mg} / \mathrm{L}$ naringenin (Additional file 1: Table S1). The total flavonoids 


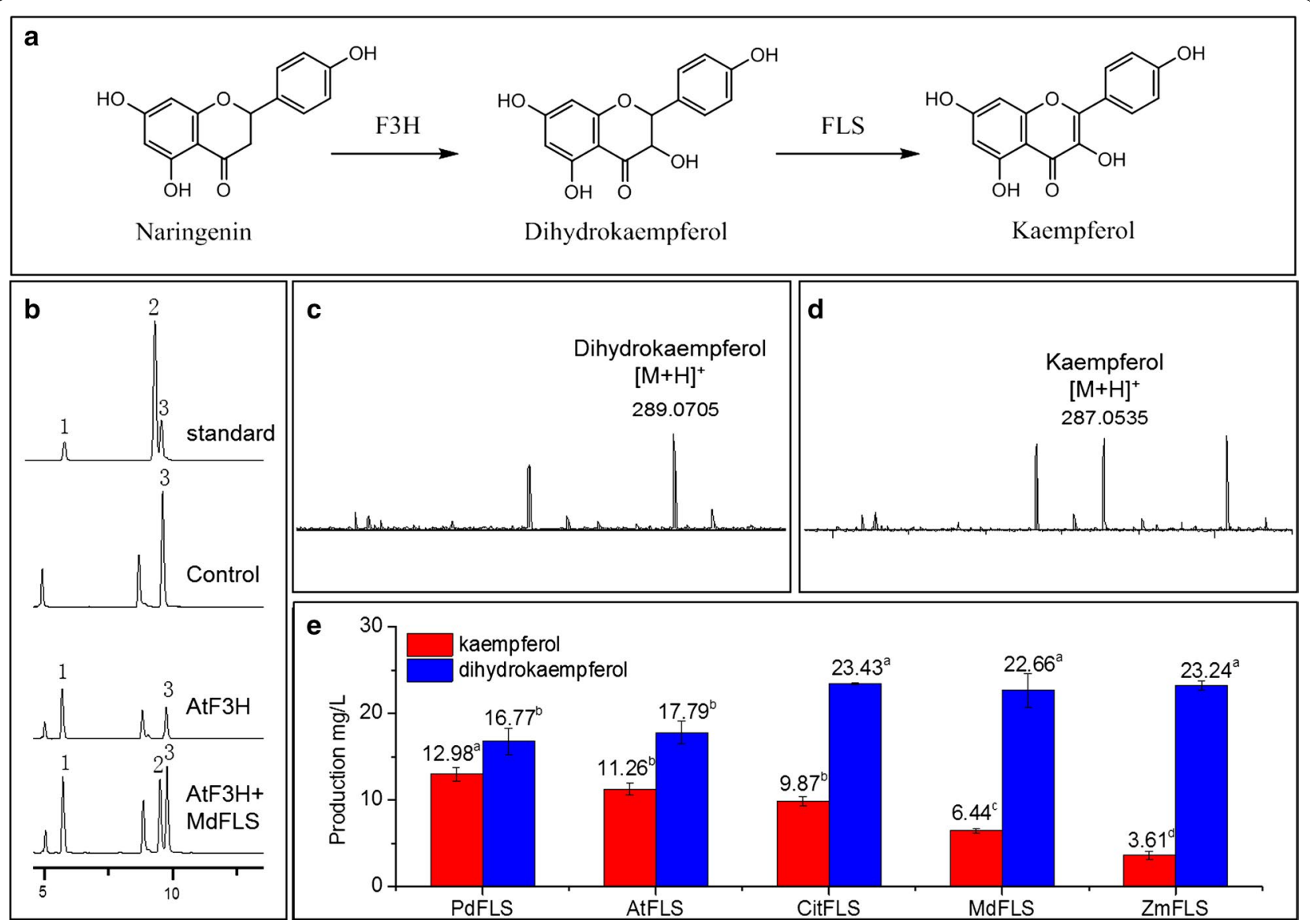

Fig. 1 Functional identification of two key enzymes of F3H and FLS in kaempferol biosynthetic pathway. a The biosynthetic pathway from naringenin to kaempferol. Naringenin is converted into dihydrokaempferol with flavanone $3 \beta$-hydroxylase $(\mathrm{F} 3 \mathrm{H})$, and dihydrokaempferol is converted into kaempferol via flavonol synthase (FLS). b HPLC analysis of the product in whole cell catalysis with overexpression of F3H and FLS in yeast. Chromatogram data were collected at $335 \mathrm{~nm}$. Peak 1, (+)-dihydrokaempferol; peak 2, kaempferol; peak 3, (2S)-naringenin. (2S)-naringenin was the substrate and was detected in the wild type W3-Y22. c LC-MS analysis of dihydrokaempferol produced by W3-AtF3H in whole cell catalysis. $\mathbf{d}$ LC-MS analysis of kaempferol produced by W3-MdFLS in whole cell catalysis. e Production of kaempferol and dihydrokaempferol of recombinant yeast strain expressing FLS from five plant species. Recombinant yeast strain expressing AtF3H and FLS from different species were fermented. Data are shown as average in triplicate assays. Error bar shows standard division. Different superscript letter indicates significant difference in Tukey analysis $(a=0.05)$

production was largely improved by introducing kaempferol biosynthetic pathway (Additional file 1: Table S1). This is probably because the expression of PdFLS and AtF3H strengthened the driving force toward flavonoid synthesis from glucose. Besides, the higher kaempferol production compared to dihydrokaempferol in W3NPFF also indicated that PdFLS is efficient in kaempferol production.

\section{Metabolic engineering for kaempferol production}

In order to further improve the production of kaempferol, we proposed to enhance the metabolic pathway of precursors. Increasing the intracellular acetyl-CoA pool and (or) malonyl-CoA pool was beneficial for high-level flavonoids production $[14,25,26]$. The biosynthetic pathway of malonyl-CoA from ethanol consists of four steps, catalyzed by alcohol dehydrogenase, aldehyde dehydrogenase, acetyl-coA synthetase and acetyl-CoA carboxylase (encoded by $A D H 2, A L D 6, A C S^{S E}$ and $A C C 1$, respectively Fig. 3a). To increase endogenous supply of acetyl-CoA or malonyl-CoA, biosynthetic genes were overexpressed in the kaempferol producing strain W3NP-FF. The gene cluster harboring $A D H 2, A L D 6$, and $A C S^{S E}$ expression cassettes (Additional file 1: Figure $\mathrm{S} 3 \mathrm{~B}$ ), and another harboring $A D H 2, A L D 6, A C S^{S E}$ and $A C C 1$ (Additional file 1: Figure $\mathrm{S} 3 \mathrm{C}$ ) expression cassettes were separately transformed to W3NP-FF, generating W3NP-FF-A3 and W3NP-FF-A4, respectively. In batch fermentation, 


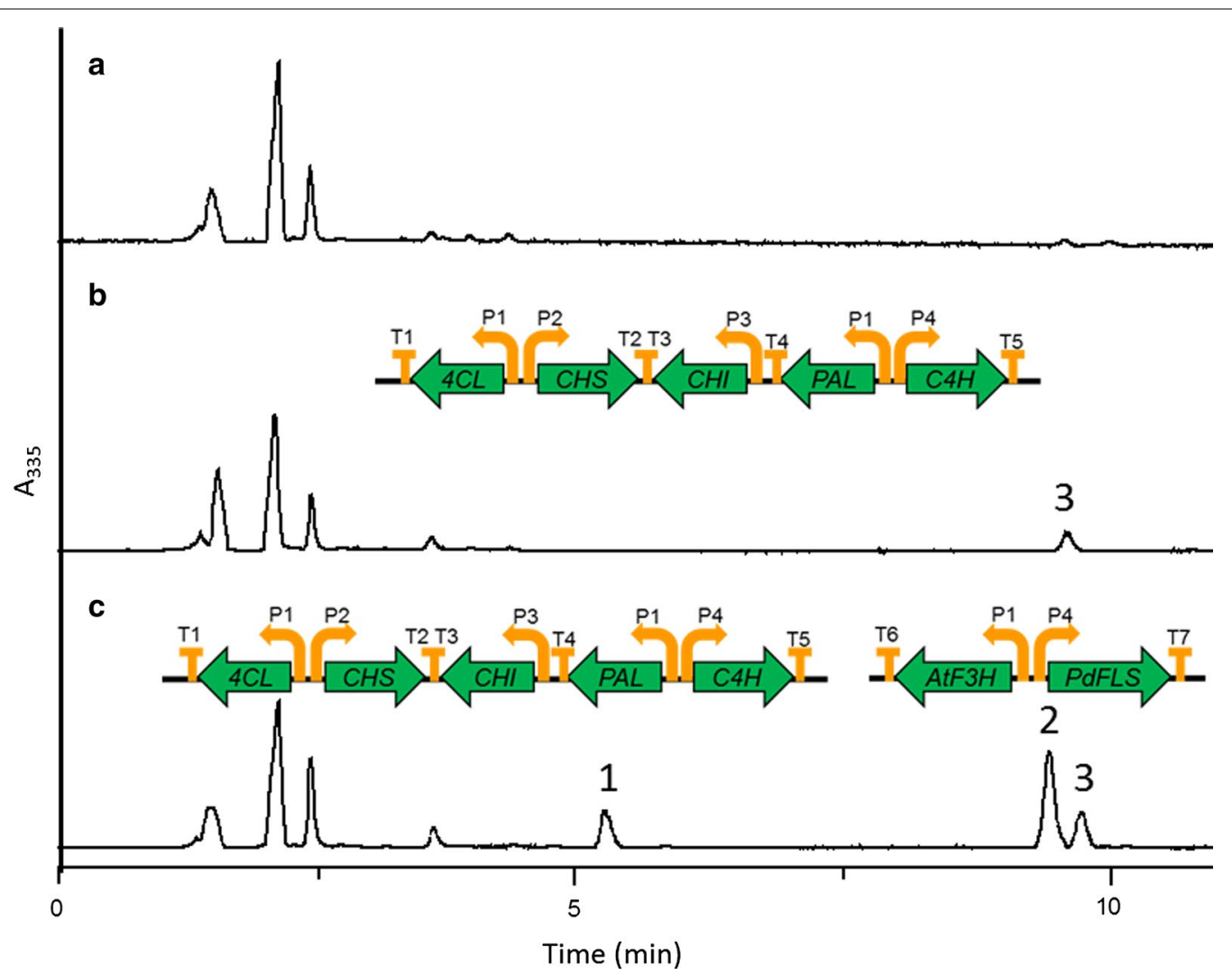

Fig. 2 Pathway construction for de novo biosynthesis of kaempferol in yeast. HPLC analysis of the broth fermented by W303-1A (a), W3NP (b) and W3NP-FF (c) from glucose. W303-1A, wild type; W3NP, a recombinant harboring the pathway of naringenin biosynthesis; W3NP-FF, a recombinant harboring the pathway of kaempferol biosynthesis. Peak 1, dihydrokaempferol; peak 2, kaempferol; peak 3, naringenin. Organization of expressed gene clusters are shown at the top of the chromatogram. P1, ADH1 promoter; P2, HXT7 promoter; P3, PGI1 promoter; P4, TDH3 promoter; T1, TPI1 terminator; T2, TPG1 terminator; T3, ADH1 terminator; T4, FBA1 terminator; T5 PDC1 terminator; T6, RPS2 terminator; T7, TDH1 terminator

W3NP-FF-A3 produced $8.6 \mathrm{mg} / \mathrm{L}$ kaempferol from glucose, which increased $23 \%$ compared to W3NPFF (Fig. 3b and Additional file 1: Table S1). However, W3NP-FF-A4 significantly decreased the production of kaempferol by $48 \%$, and also decreased the production of naringenin and dihydrokaempferol by 75 and $63 \%$ respectively, compared to W3NP-FF (Fig. $3 \mathrm{~b}$ and Additional file 1: Table S1). Besides, the ACC1 overexpression caused a $33 \%$ reduction in final $\mathrm{OD}_{600}$, and reduce by $37 \%$ in specific kaempferol production compared to these of W3NP-FF-A3 in fermentation from glucose (Fig. 3b and Additional file 1: Table S1).

ACC1 encodes cytosolic acetyl-CoA carboxylase (ACCase), catalyzing the malonyl-CoA formation from acetyl-CoA in S. cerevisiae, which is regarded as the rate limiting enzyme in fatty acid synthesis, and is thus tightly regulated in transcription and post-translation levels [27, 28]. In most case, overexpressing a native or a mutant $A C C 1$ increased the malonyl-CoA pool or the derived products [29-32]. By contrast, some studied suggested that the ACCase overexpression impaired cell growth [29, 32, 33], or did not promote the production of malonyl-CoA derived products significantly $[34,35]$. The reasons for this phenomenon have generally attributed to an imbalanced synthesis of long-chain fatty acids, depletion of intermediates or high metabolic burden [27, 29, 32]. In this study, an $A C C 1$ mutant with Ser659Ala and Ser1157Ala, which improves ACCase activity by abolishing Snf1-dependent regulation, was driven by a PGK1 promoter. Thus, expression of ACCase had been enhanced in both transcription and post-translation levels. The excessively increased ACCase activity may cause imbalanced synthesis of long-chain fatty acids and/or abnormal intracellular levels of acetyl-CoA and AMP, and interfere cell growth and metabolism, including flavonoids synthesis (Fig. 3b and Additional file 1: Table S1). Our results suggest that a simple and straightforward ACCase overexpression could not increase malonyl-CoA derived products as expected, and introducing a dynamic ACCase regulation or heterologous malonyl-CoA synthesis pathway would be a more promising strategy $[36,37]$. 

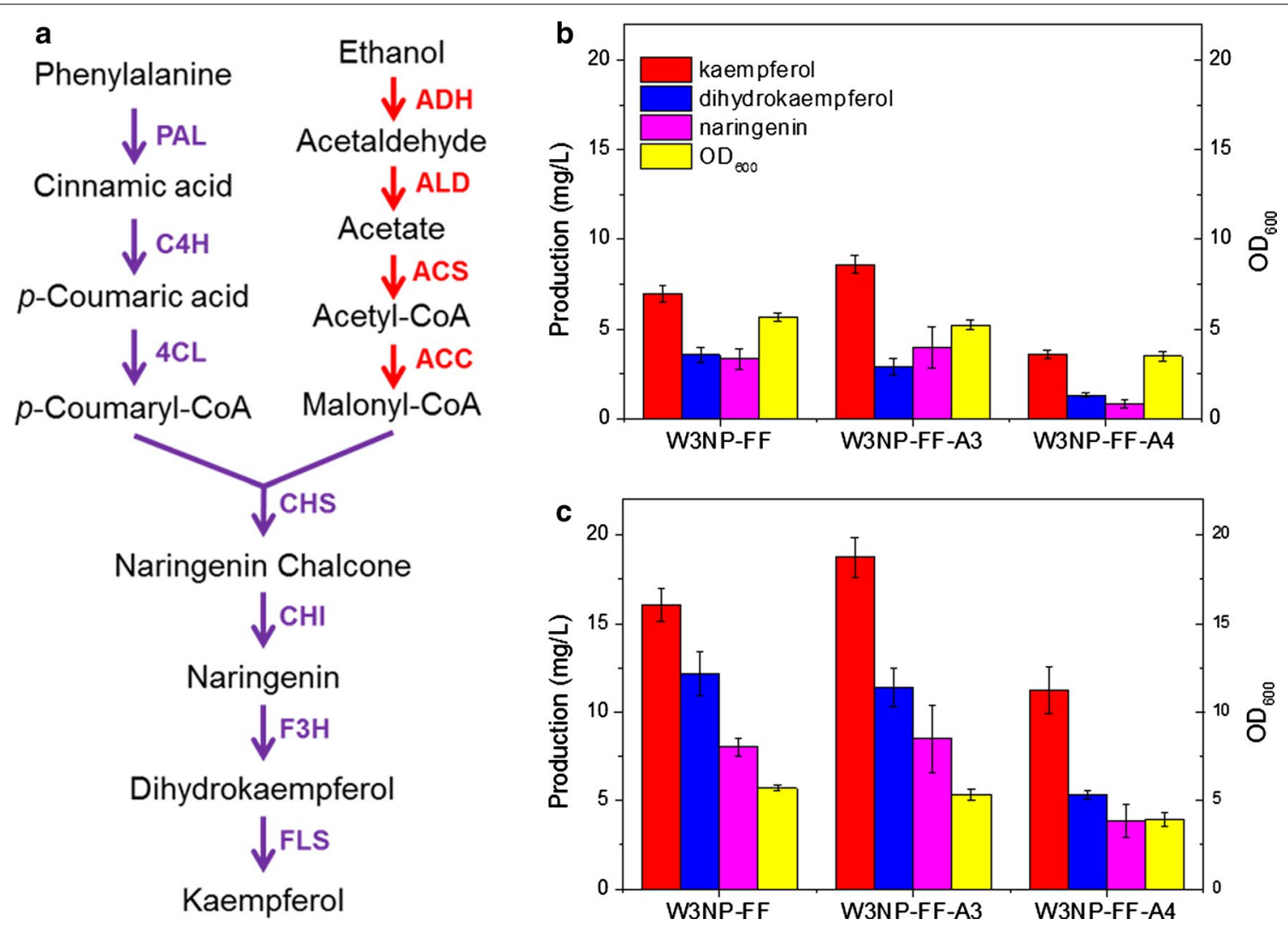

Fig. 3 Metabolic engineering for high kaempferol production in S. cerevisiae. a Metabolic pathway and engineering scheme for kaempferol production. Purple arrows represent reactions catalyzed by exogenous enzymes from plant in kaempferol synthesis pathway. Red arrows represent reactions catalyzed by endogenous enzymes in yeast, which are involved in the precursor synthesis, and overexpressed for kaempferol production. PAL, phenylalanine ammonia lyase; $\mathrm{C4H}$, cinnamic acid 4-hydroxylase; $4 \mathrm{CL}$, 4-coumaric acid ligase; $\mathrm{CHS}$, chalcone synthase; $\mathrm{CHI}$, chalcone isomerase; F3H, flavanone 3ß-hydroxylase; FLS, flavonol synthase; ADH, alcohol dehydrogenase; ALD, aldehyde dehydrogenase; ACS, acetyl-coA synthetase; ACC, acetyl-CoA carboxylase. $\mathbf{b} \mathrm{OD}_{600}$ and production of naringenin, dihydrokaempferol and kaempferol in batch fermentation at $60 \mathrm{~h}$. Glucose

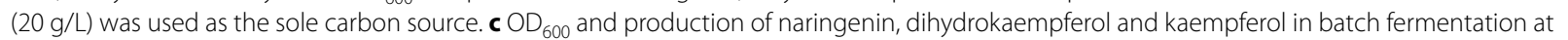
$60 \mathrm{~h}$. The media were supplemented with $20 \mathrm{~g} / \mathrm{L}$ glucose and $1 \mathrm{mM}$ p-coumarate. Data are shown as average \pm standard division in triplicate tests

Another important precursor of flavonoids biosynthesis is phenylalanine. The intracellular L-phenylalanine synthesis is tightly regulated in S. cerevisiae $[10,38]$. Here, we supplemented $p$-coumarate in the fermentation medium in order to partially alleviate the limitation of precursor supply [10,38]. The $p$-coumarate supplement significantly increased total flavonoids production by about 2-3.5 times for W3NP-FF, W3NP-FF-A3 and W3NP-FF-A4 (Fig. 3b, c and Additional file 1: Table S1). Particularly, the kaempferol production of W3NP-FFA3 reached $18.76 \mathrm{mg} / \mathrm{L}$, which is close to the highest reported value $(22.57 \mathrm{mg} / \mathrm{L})$ [39].

\section{Fermentation optimization for kaempferol production}

Fermentation conditions, such as $\mathrm{pH}$, media components, temperature, stirring speed and ventilation rate, also largely affect the titer of products in microbial cell factory. In this research, a Quasi exponent feed strategy [40] was adopted in fed-batch fermentation for high kaempferol production with W3NP-FF and W3NP-FFA3. The $\mathrm{OD}_{600}$ and the production of flavonoids were measured and shown in Fig. 4a, b. Both of W3NP-FF and W3NP-FF-A3 produced much higher kaempferol in fed-batch fermentation than that in batch fermentation (Figs. 3b, c, and 4a, b). To increase substrate supply, $1 \mathrm{mM} p$-coumarate was supplemented after $24 \mathrm{~h}$ fermentation. Then the production of kaempferol, dihydrokaempferol and naringenin were consequently increased for both W3NP-FF-A3 and W3NP-FF (Fig. 4a, b). For W3NP-FF-A3, the kaempferol production continuously increased in the early $40 \mathrm{~h}$ (Fig. 4b); while for W3NP$\mathrm{FF}$, the kaempferol production reached the top at $32 \mathrm{~h}$, but with a stationary phase from 12 to $24 \mathrm{~h}$ (Fig. 4a). This result showed that overexpression of acetyl-CoA 

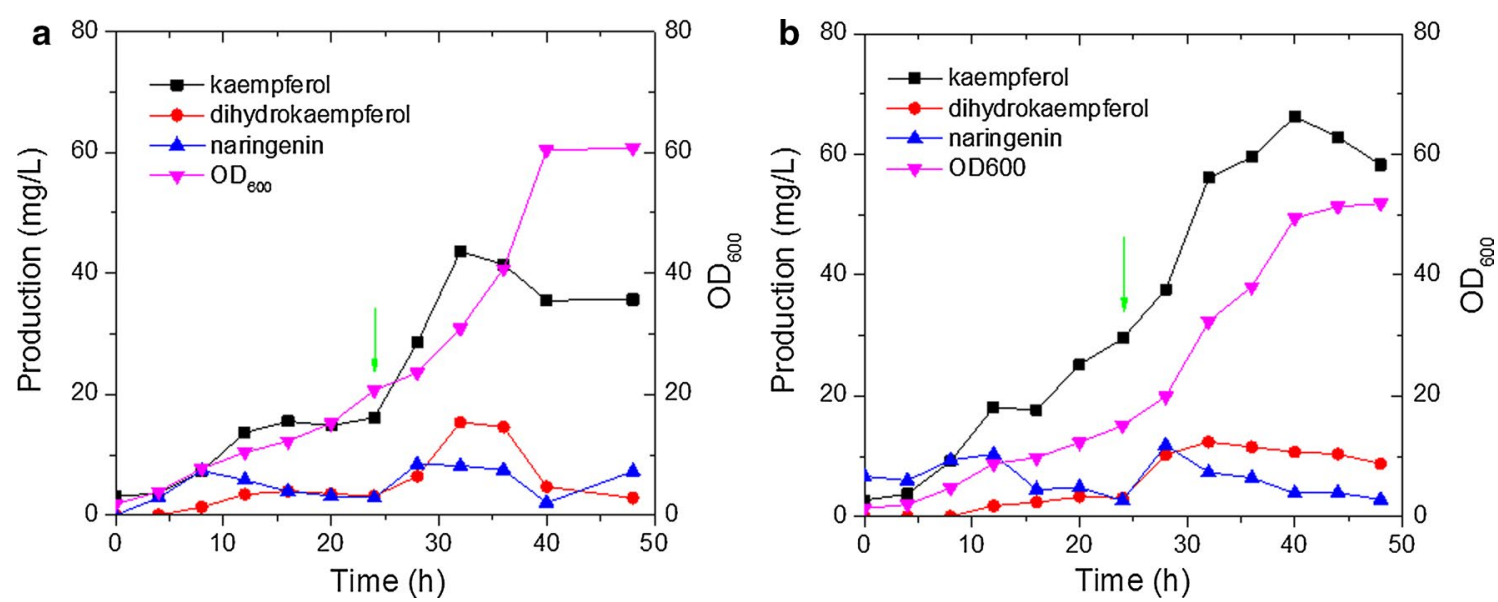

Fig. 4 Fed-batch fermentation of kaempferol in well controlled fermenters. $\mathrm{OD}_{600}$ and production of naringenin, dihydrokaempferol and kaempferol are shown in fed-batch fermentation with W3NP-FF (a) and W3NP-FF-A3 $(\mathbf{b})$. The fermentations were performed at $30^{\circ} \mathrm{C}$, and pH was maintained at 5.0. The feed was started when residual ethanol and glucose were completely depleted. A Quasi exponent feed strategy was adopted. Green arrow indicates $1 \mathrm{mM} \mathrm{p}$-coumarate was supplemented at $24 \mathrm{~h}$

synthetic pathway ( $A D H 2, A L D 6$, and $\left.A C S^{S E}\right)$ not only increased the titer of kaempferol, but also improved the persistence of kaempferol production. The highest kaempferol titer in fed-batch fermentation achieved $66.29 \mathrm{mg} / \mathrm{L}$ by W3NP-FF-A3, which is 3.5 -fold over that in batch fermentation (Fig. 4b, Additional file 1: Table S1 and S2). To our knowledge, it is about 2.5 times of the reported highest titer $(26.57 \mathrm{mg} / \mathrm{L}$ kaempferol produced by an engineered S. cerevisiae [39]).

In the flavonoid synthetic pathway, malonyl-CoA and $p$-coumaroyl-CoA are two direct precursors in chalcone forming. In this research, the intracellular supply of malonyl-CoA has been enhanced by over-expression the acetyl-CoA synthetic pathway; on the other hand, the supply of $p$-coumaroyl-CoA has been satisfied by supplementing $p$-coumarate in the medium, which trends to add extra cost in fermentation. Alternatively, it has been reported that engineering the aromatic amino acid biosynthesis pathway and overexpressing the tyrosine ammonia-lyase (TAL) increased the formation and accumulation of $p$-coumaric acid in yeast cells [41]. This is an attractive strategy for promoting the production of $p$-coumaric acid derived products from glucose, and saving $p$-coumaric acid supplement in fermentation. Recently, Rodriguez et al. expressed $4 C L$, $\mathrm{CHS}, \mathrm{CHI}, \mathrm{CHR}$ (encoding chalcone reductase), F3H (from Astragalus mongholicus), FLS (from Arabidopsis thaliana) and CPR (encoding cytochrome $\mathrm{P} 450$ reductase) and FMO (encoding a cytochrome P450 flavonoid monooxygenases) genes in $p$-coumaric acid over-accumulated strains, to produce several flavonoids including kaempferol [39]. Taking advantage of the enhanced $p$-coumaric acid synthesis, the engineered strain produced $26.57 \mathrm{mg} / \mathrm{L}$ kaempferol [39], which is the highest reported titer to our best knowledge. In this research, we comprehensively applied pathway construction, enzyme selection, metabolic engineering, intermediate supplement and fermentation optimization to realize and upgrade kaempferol production gradually, and achieved the highest kaempferol production at $66.29 \mathrm{mg} / \mathrm{L}$. Here, we emphasize the combination of multi biological solutions for efficient product synthesis in a microbial cell factory.

\section{Conclusions}

In this study, we successfully synthesized kaempferol through $S$. cerevisiae recombinants expressing FLS from $P$. deltoides, which resulted in higher kaempferol production than those from A. thaliana, C. unshiu, M. domestica, and Z. mays. Through expressing PAL, C4H, 4CL, CHS, CHI, AtF3H and PdFLS, we constructed a yeast recombinant that synthesizes kaempferol de novo. Furthermore, we demonstrated that overexpressing the acetyl-CoA synthetic pathway (consisted of $A D H 2, A L D 6$ and $A C S^{S E}$ ) in cytoplasm and $p$-coumarate supplement in media would significantly increase kaempferol titer of the recombinant. Fermentation conditions are also closely related to the kaempferol biosynthesis that fed-batch fermentation in a bioreactor with a Quasi exponent feed strategy largely improved kaempferol production compared to batch fermentation in flask. The titer of kaempferol reached up to $66.29 \mathrm{mg} / \mathrm{L}$ after $40 \mathrm{~h}$ fermentation. 
To our knowledge, it is the highest kaempferol titer in microbial cell factories currently.

\section{Methods}

\section{Genes and strains}

Flavonol synthases from $A$. thaliana (accessing number NP_196481.1, encoded by AtFLS), C. unshiu (accessing number: Q9ZWQ9.1, encoded by CitFLS), M. domestica (accessing number: NP_001306179.1, encoded by MdFLS),Z. Mays (accessing number: XP_008646309.1, encoded by $Z m F L S$ ) and $P$. deltoides (TIGR accession number: TC74233 [23], encoded by PdFLS) were investigated in this work. Flavanone $3 \beta$-hydroxylase (F3H) was from $A$. thaliana (encoded by $A t F 3 H$, accessing number: NP_190692.1). PAL, C4H, 4CL, CHS and CHI were from Erigeron breviscapus (Vant.) Hand-Mazz. (kindly provided by Guang-Hui Zhang [42]). The codon usage of CitFLS, MdFLS, ZmFLS, PdFLS, PAL, C4H, 4CL, CHS and CHI were optimized for S. cerevisiae (Additional file 1: Table S3) and synthesized by a local company.

Escherichia coli DH5 $\alpha$ was used for gene cloning. S. cerevisiae W303-1A was used for genetic engineering and kaempferol production. All strains and plasmids used in this study are shown in Table 1.

\section{Gene cloning and plasmid construction}

All DNA manipulations were performed according to standard procedures [43]. Phusion High-Fidelity DNA Polymerase (Thermo Fisher Scientific) was used for PCR amplification. A DNA fragment, named Ter-4, was designed for Golden gate cloning, which contained two terminators $\left(T_{R S P 2}\right.$ and $\left.T_{T D H 1}\right)$, being separated by two $B s a \mathrm{I}$ digest sites (the scheme was shown in Additional file 1: Figure S3A). The Ter-4 fragment was synthesized by a local company (Synbio Technologies), and cloned to pUC57. Then the Ter-4 fragment and YCplac22 were amplified (primers: T4-YCP-F/T4-YCP-R, Y22-T4-F/ Y22-T4-R, Additional file 1: Table S4) and assembled together using CPEC (Circular Polymerase Extension Cloning [44]), generating Y22-T4 (Additional file 1: Figure S4), the backbone for FLS and F3H expression. In CPEC, the vector and the insert share overlapping regions at the ends, and the hybridized insert and vector extend using each other as a template until they complete a full circle in a PCR system, and finally the PCR product is transformed directly to DH5 $\alpha$. For assembly of gene expression cassettes, 3 DNA fragments, including a head-to-head promoter fragment, AtF $3 H$ and each of the FLS genes (AtFLS, CitFLS, MdFLS, PdFLS, ZmFLS), were

Table 1 Plasmids and strains used in this study

\begin{tabular}{|c|c|c|}
\hline Plasmid or strain & Genotype & Source or reference \\
\hline \multicolumn{3}{|l|}{ Plasmids } \\
\hline YCplac22 & Amp Trp1 & Gietz [50] \\
\hline YCplac33 & AmpURA3 & Gietz [50] \\
\hline Y22-T4 & $\mathrm{T}_{A D H 1} / \mathrm{T}_{T D H 3}$ & This work \\
\hline Y22-AtF3H & $\mathrm{P}_{P G K 1}-A t F 3 H-T_{A D H 1}$ & This work \\
\hline Y22-T4-AtF3H-AtFLS & $\mathrm{P}_{P G K 1}-A t F 3 H-T_{A D H 1} / P_{T D H 3}-A t F L S-T_{T D H 1}$ & This work \\
\hline Y22-T4-AtF3H-CitFLS & $\mathrm{P}_{\mathrm{PGK1}}-A t F 3 H-\mathrm{T}_{A D H 1} / \mathrm{P}_{\mathrm{TDH} 3}-\mathrm{CitFLS- \textrm {T } _ { T D H 1 }}$ & This work \\
\hline Y22-T4-AtF3H-MdFLS & $\mathrm{P}_{P G K 1}-A t F 3 H-T_{A D H 1} / P_{T D H 3}-M d F L S-T_{T D H 1}$ & This work \\
\hline Y22-T4-AtF3H-PdFLS & $\mathrm{P}_{P G K 1}-A t F 3 H-T_{A D H 1} / P_{T D H 3}-P d F L S-T_{T D H 1}$ & This work \\
\hline Y22-T4-AtF3H-ZmFLS & $\mathrm{P}_{P G K 1}-A t F 3 H-T_{A D H 1} / P_{T D H 3}-Z m F L S-T_{T D H 1}$ & This work \\
\hline Y33-ALAC-ADH2 & $P_{\text {TEF } 2}-A C S^{S E}-T_{R P S 2} / P_{P G K 1}-A L D 6-T_{T D H 1} / P_{H X T 7}-A D H 2-T_{R P L 9 A}$ & This work \\
\hline Y33-ALAC-ACAD & $\mathrm{P}_{\text {TEF2 }}-A C S^{S E}-\mathrm{T}_{\text {RPS2 } 2} / \mathrm{P}_{P G K 1}-A L D 6-\mathrm{T}_{T D H 1} / \mathrm{P}_{H X T 7}-\mathrm{ADH} 2-\mathrm{T}_{\text {RPL9A }} / \mathrm{P}_{P G K 1}-A C C 1-\mathrm{T}_{C C W 12}$ & This work \\
\hline \multicolumn{3}{|c|}{ 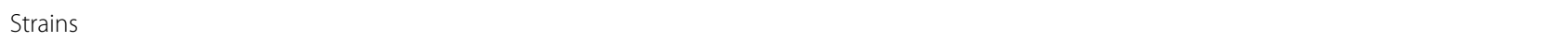 } \\
\hline W303-1A & MATa leu2-3112 ura3-1 trp1-92 his3-11,15 ade2-1 can 1-100 & Thomas [51] \\
\hline W3-AtF3H & W303-1A carrying Y22-AtF3H & This work \\
\hline W3-AtFLS & W303-1A carrying Y22-T4-AtF3H-AtFLS & This work \\
\hline W3-CitFLS & W303-1A carrying Y22-T4-AtF3H-CitFLS & This work \\
\hline W3-MdFLS & W303-1A carrying Y22-T4-AtF3H-MdFLS & This work \\
\hline W3-PdFLS & W303-1A carrying Y22-T4-AtF3H-PdFLS & This work \\
\hline W3-ZmFLS & W303-1A carrying Y22-T4-AtF3H-ZmFLS & This work \\
\hline W3NP & YORWdelta17::His3/P $\mathrm{P}_{A D H 1}-4 C L-\mathrm{T}_{T P 11} / \mathrm{P}_{H X T 7}-\mathrm{CHS}-\mathrm{T}_{T P G 1} / \mathrm{P}_{P G 11}-\mathrm{CHI}-\mathrm{T}_{A D H 1} / \mathrm{P}_{A D H 1}-\mathrm{PAL}-\mathrm{T}_{\mathrm{FBA1}} / \mathrm{P}_{\mathrm{TDH}}-\mathrm{C} 4 \mathrm{H}-\mathrm{T}_{P D C 1}$ & This work \\
\hline W3NP-FF & W3NP carrying Y22-T4-AtF3H-PdFLS and YCplac33 & This work \\
\hline W3NP-FF-A3 & W3NP carrying Y22-T4-AtF3H-PdFLS and Y33-ALAC-ADH2 & This work \\
\hline W3NP-FF-A4 & W3NP carrying Y22-T4-AtF3H-PdFLS and Y33-ALAC-ACAD & This work \\
\hline
\end{tabular}


amplified (primers: P1-F/P1-R for promoter fragment, AtF3H-GG2-F/AtF3H-GG2-R for AtF3H, FLS-GG1-F/ FLS-GG1-R for each FLS gene, Additional file 1: Table S4) respectively, and ligated to Y22-T4 through golden gate cloning [45], generating $\mathrm{FLS}$ and $\mathrm{F} 3 \mathrm{H}$ expression cassettes (Additional file 1: Figures S3A, S4). Thus, Y22-T4-F3H-AtFLS, Y22-T4-AtF3H-CitFLS, Y22T4-AtF3H-MdFLS, Y22-T4-AtF3H-PdFLS and Y22T4-AtF3H-ZmFLS was constructed. Then, expression cassette of $A t F 3 H$ was amplified (primer: F3H-CPF/F3HCPR, Additional file 1: Table S4) and ligated to YCplac22 through CPEC [44], resulting in Y22-AtF3H. Similarly, expression cassettes of $A L D 6, A C S^{S E}$ [46], $A D H 2$ and ACC1 (a mutant with Ser659Ala and Ser1157Ala, [29]) were constructed through golden gate cloning. Then, the cassettes of $A L D 6, A C S^{S E}$ and $A D H 2$ were inserted into YCplac33 through Gibson assembling [47] (primers: Y33-F/Y33-R, ALAC-GibF/ALAC-GibR, ADH2-GibF/ ADH2-GibR, Additional file 1: Table S4), resulting in the plasmid Y33-ALAC-ADH2 (Additional file 1: Figure S3B). Cassettes of $A L D 6, A C S^{S E}, A D H 2$ and $A C C 1$ were inserted into YCplac33 through Gibson assembling [47] (primers: Y33-F/Y33-R, ALAC-GibF/ALAC-GibR, ACAD-GibF/ADH2-GibR, Additional file 1: Table S4), generating the plasmid Y33-ALAC-ACAD (Additional file 1: Figure S3C).

\section{Strain construction}

To generate a naringenin-producing $S$. cerevisiae recombinant, a modularized two-step (M2S) chromosome integration technique [45] was applied for $P A L, C 4 H$, $4 C L, C H S$ and $C H I$ expression. In brief, $P A L$ and $C 4 H$; $4 C L$ and $C H S$; and $C H I$ was ligated with promoters and terminators respectively through golden gate cloning, forming the expression cassettes. Then, DNA fragments harboring these cassettes were transformed together with His marker and homologous arms into wild strain (W303-1A), and integrated into genome through DNA assembler [48] (Additional file 1: Figure S3D), resulting in the naringenin-producing recombinant, W3NP. Then, Y22-T4-AtF3H-PdFLS was co-transformed into W3NP with YCplac33, Y33-ALAC-ADH2 and Y33-ALACACAD respectively, resulting in W3NP-FF, W3NP-FF-A3 and W3NP-FF-A4.

\section{Media and culture condition}

Escherichia coli was grown in Luria-Bertani (LB) medium at $37{ }^{\circ} \mathrm{C}$. Ampicillin $(50 \mu \mathrm{g} / \mathrm{mL})$ was added to the medium when required. Yeast strains were grown at $30{ }^{\circ} \mathrm{C}$ in YPD medium $(10 \mathrm{~g} / \mathrm{L}$ yeast extract, $20 \mathrm{~g} / \mathrm{L}$ Bacto peptone, and $20 \mathrm{~g} / \mathrm{L}$ glucose) or defined mineral medium (YSCD), containing $6.7 \mathrm{~g} / \mathrm{L}$ yeast nitrogen base (YNB) without amino acids (Difco, Detroit, Michigan), supplemented with the appropriate auxotrophic requirements and $20 \mathrm{~g} / \mathrm{L}$ glucose. YSCD supplemented with $5 \mathrm{mM}$ sodium ascorbate and $0.1 \mathrm{mM} \mathrm{Fe}_{2} \mathrm{SO}_{4}$ was used for batch fermentation in flask, and $1 \mathrm{mM} p$-coumarate was added when required.

The medium for fed-batch fermentation contained: $20 \mathrm{~g} / \mathrm{L}$ glucose, $15 \mathrm{~g} / \mathrm{L}\left(\mathrm{NH}_{4}\right)_{2} \mathrm{SO}_{4}, 8 \mathrm{~g} / \mathrm{L} \mathrm{KH}_{2} \mathrm{PO}_{4}$, $6.2 \mathrm{~g} / \mathrm{L} \mathrm{MgSO}_{4} \cdot 7 \mathrm{H}_{2} \mathrm{O}, 12 \mathrm{~mL} / \mathrm{L}$ vitamin solution and $10 \mathrm{~mL} / \mathrm{L}$ trace metal solution, where the vitamin solution contained $0.05 \mathrm{~g} / \mathrm{L}$ biotin, $1 \mathrm{~g} / \mathrm{L}$ calcium pantothenate, $1 \mathrm{~g} / \mathrm{L}$ nicotinic acid, $25 \mathrm{~g} / \mathrm{L}$ inositol, $1 \mathrm{~g} / \mathrm{L}$ thiamine $\mathrm{HCl}, 1 \mathrm{~g} / \mathrm{L}$ pyridoxal $\mathrm{HCl}, 0.2 \mathrm{~g} / \mathrm{L} p$-aminobenzoic acid and $2.5 \mathrm{~g} / \mathrm{L}$ adenine; the trace metal solution contained: $5.75 \mathrm{~g} / \mathrm{L} \mathrm{ZnSO}_{4} \cdot 7 \mathrm{H}_{2} \mathrm{O}, 0.32 \mathrm{~g} / \mathrm{L} \mathrm{MnCl}_{2} \cdot 4 \mathrm{H}_{2} \mathrm{O}$, $0.47 \mathrm{~g} / \mathrm{L} \mathrm{CoCl} 2 \cdot 6 \mathrm{H}_{2} \mathrm{O}, 0.48 \mathrm{~g} / \mathrm{L} \mathrm{Na}_{2} \mathrm{MoO}_{4} \cdot 2 \mathrm{H}_{2} \mathrm{O}, 2.9 \mathrm{~g} / \mathrm{L}$ $\mathrm{CaCl}_{2} \cdot 2 \mathrm{H}_{2} \mathrm{O}, 2.8 \mathrm{~g} / \mathrm{L} \mathrm{FeSO}_{4} \cdot 7 \mathrm{H}_{2} \mathrm{O}$ and $80 \mathrm{~mL} / \mathrm{L} 0.5 \mathrm{M}$ EDTA, pH 8.0. The same medium was used for seed culture in fed-batch fermentation [49].

\section{Fermentation \\ Whole cell catalysis}

Inoculum was cultured in YSCD medium at $30{ }^{\circ} \mathrm{C}$ for $12 \mathrm{~h}$. The pre-culture was refreshed in $20 \mathrm{~mL}$ YSCD to $\mathrm{OD}_{600}=1$. Cells were then collected and re-suspended in $3 \mathrm{~mL}$ YSCD supplemented with $5 \mathrm{mM}$ sodium ascorbate, $0.1 \mathrm{mM} \mathrm{Fe}_{2} \mathrm{SO}_{4}$ and $100 \mathrm{mg} / \mathrm{L}(2 \mathrm{~S}$ )-naringenin (Solarbio Life Sciences), to a final cell concentration of $\mathrm{OD}_{600}=5$. The reaction was incubated at $30{ }^{\circ} \mathrm{C}$ in an orbital shaker (220 rpm) for $24 \mathrm{~h}$.

\section{Batch fermentation}

Inoculum was cultured in YSCD medium at $30{ }^{\circ} \mathrm{C}$ for $12 \mathrm{~h}$. The pre-culture was then used to inoculate $20 \mathrm{~mL}$ batch fermentation medium (YSCD supplemented with $5 \mathrm{mM}$ sodium ascorbate and $0.1 \mathrm{mM} \mathrm{Fe}_{2} \mathrm{SO}_{4}$ ) in $250 \mathrm{~mL}$ shaker flasks to a final cell concentration of $\mathrm{OD}_{600}=1$. One $\mathrm{mM} p$-coumarate was added as precursor when required. The fermentation lasted for $60 \mathrm{~h}$.

\section{Fed-batch fermentation}

Glycerol-stocked cells were inoculated into $40 \mathrm{~mL}$ YSCD and cultured at $30{ }^{\circ} \mathrm{C}, 220 \mathrm{rpm}$ for $24 \mathrm{~h}$. The culture was transferred to $360 \mathrm{~mL}$ fed-batch fermentation medium, and cultured for another $24 \mathrm{~h}$. $300 \mathrm{~mL}$ seeds were inoculated to $1.5 \mathrm{~L}$ fed-batch fermentation medium in Baoxin bioreactor with a maximal working volume of $3 \mathrm{~L}$. The fermentations were performed at $30{ }^{\circ} \mathrm{C}$, and $\mathrm{pH}$ was maintained at 5.0 with automatic addition of ammonium hydroxide or $1 \mathrm{M} \mathrm{H}_{2} \mathrm{SO}_{4}$. The agitation rate was kept between 300 and $800 \mathrm{rpm}$, and the air flow was set as $1.5 \mathrm{vvm}$. The dissolved oxygen concentration was controlled above $40 \%$ throughout regulation the agitation rate [40]. The feed was started after residual ethanol 
and glucose were completely depleted. A Quasi exponent feed strategy was adopted as described [40]. Feed reagent contained: $386 \mathrm{~g} / \mathrm{L}$ glucose, $9 \mathrm{~g} / \mathrm{L} \mathrm{KH}_{2} \mathrm{PO}_{4}, 5.12 \mathrm{~g} / \mathrm{L}$ $\mathrm{MgSO}_{4} \cdot 7 \mathrm{H} 2 \mathrm{O}, 3.5 \mathrm{~g} / \mathrm{L} \mathrm{K} \mathrm{SO}_{4}, 0.28 \mathrm{~g} / \mathrm{L} \mathrm{Na}_{2} \mathrm{SO}_{4}, 5 \mathrm{~g} / \mathrm{L}$ adenine, $12 \mathrm{~mL} / \mathrm{L}$ vitamin solution and $10 \mathrm{~mL} / \mathrm{L}$ trace metal solution. $1 \mathrm{mM} p$-coumarate was supplemented at $24 \mathrm{~h}$.

\section{Detection and quantification of the products}

Flavonoids were extracted directly from the culture with an equal volume of methanol. The extraction were analyzed by High Performance Liquid Chromatography (HPLC, Agilent), using Phenomenex Kinetex Biphenyl Column $(5 \mu \mathrm{m}, 250 \times 4.6 \mathrm{~mm})$ equipped with a photodiode array detector. The mobile phase consisted of acetonitrile and water $(0.1 \%$ phosphoric acid) using a gradient elution of $30-40 \%$ acetonitrile for $10 \mathrm{~min}, 40-95 \%$ acetonitrile for $2.5 \mathrm{~min}, 95-30 \%$ acetonitrile for $2.5 \mathrm{~min}$ and $30 \%$ acetonitrile for $5 \mathrm{~min}$, at flow rate of $1 \mathrm{~mL} /$ min. Samples were analyzed by LC-MS using a Thermo U3000-LTQ XL (Thermo Scientific) system coupled to the ion trap mass spectrometer with an ESI source operating in the positive mode. LC-MS analysis was operated with the same LC operation method, except $0.1 \%$ formic acid was substitute to phosphoric acid. Quantification of the kaempferol was based on the peak areas of absorbance at $335 \mathrm{~nm}$. Quantification of dihydrokaempferol and naringenin was based on the peak areas of absorbance at $290 \mathrm{~nm}$.

\section{Additional file}

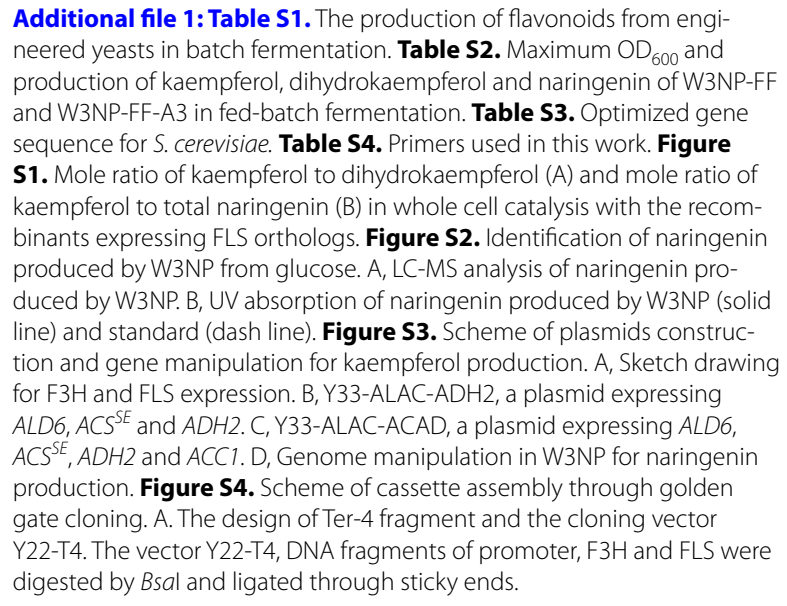

\section{Authors' contributions}

LD and WD contributes equally in this work. WD, JC and $\mathrm{HJ}$ conceived and designed the manuscript. LD and WD performed the experimental research and drafted the manuscript. XL constructed the recombinant W3NP. XC carried out the fed-batch fermentation. $\mathrm{HJ}, \mathrm{EH}$ and JC outlined the structure and reviewed the manuscript. All authors read and approved the final manuscript.

\begin{abstract}
Author details
${ }^{1}$ Key Laboratory of Industrial Microbiology, College of Biotechnology, Tianjin University of Science \& Technology, Tianjin, China. ${ }^{2}$ Key Laboratory of Systems Microbial Biotechnology, Tianjin Institute of Industrial Biotechnology, Chinese Academy of Sciences, Tianjin, China. ${ }^{3}$ University of Chinese Academy of Sciences, Beijing, China. ${ }^{4}$ State Key Laboratory of Quality Research in Chinese Medicine, Institute of Chinese Medical Sciences, University of Macau, Macau, China.
\end{abstract}

Acknowledgements

Not applicable.

\section{Competing interests}

The authors declare that they have no competing interests.

\section{Availability of data and materials}

All data generated or analyzed during this study are included in this published article.

\section{Consent for publication}

Not applicable.

Ethics approval and consent to participate

Not applicable.

\section{Funding}

This work was supported by a 973 Program (2015CB755704), the Hundred Talent Program of the Chinese Academy of Sciences and a National Natural Science Foundation of China (31670100) to HJ, and a Tianjin Research Program of Application Foundation and Advanced Technology (15JCYBJC24200), and a National Natural Science Foundation of China (31501041) to WD, and a startup research grant from University of Macau (SRG2015-00062-ICMS-QRCM) to JC.

\section{Publisher's Note}

Springer Nature remains neutral with regard to jurisdictional claims in published maps and institutional affiliations.

Received: 1 June 2017 Accepted: 18 September 2017

Published online: 26 September 2017

\section{References}

1. Calderón-Montaño JM, Burgos-Morón E, Pérez-Guerrero C, López-Lázaro M. A review on the dietary flavonoid kaempferol. Mini Rev Med Chem. 2011;11:298.

2. Chen AY, Chen YC. A review of the dietary flavonoid, kaempferol on human health and cancer chemoprevention. Food Chem. 2013;138:2099-107.

3. Granado-Serrano AB, Martin MA, Bravo L, Goya L, Ramos S. Quercetin induces apoptosis via caspase activation, regulation of $\mathrm{BCl}-2$, and inhibition of PI-3-kinase/Akt and ERK pathways in a human hepatoma cell line (HepG2). J Nutr. 2006;136:2715-21.

4. Chandramohan G, Al-Numair KS, Alsaif MA, Veeramani C. Antidiabetic effect of kaempferol a flavonoid compound, on streptozotocin-induced diabetic rats with special reference to glycoprotein components. Prog Nutr. 2015;17:50-7.

5. Muthukrishnan SD, Kaliyaperumal A, Subramaniyan A. Identification and determination of flavonoids, carotenoids and chlorophyll concentration in Cynodon dactylon (L.) by HPLC analysis. Nat Prod Res. 2015;29:785-90.

6. Agar OT, Dikmen M, Ozturk N, Yilmaz MA, Temel H, Turkmenoglu FP. Comparative studies on phenolic composition, antioxidant, wound healing and cytotoxic activities of selected Achillea L. Species Growing in Turkey. Molecules. 2015;20:17976-8000.

7. Koopman F, Beekwilder J, Crimi B, Van HA, Hall RD, Bosch D, van Maris AJ, Pronk JT, Daran JM. De novo production of the flavonoid naringenin in engineered Saccharomyces cerevisiae. Microb Cell Fact. 2012;11:155. 
8. Zhu S, Wu J, Du G, Zhou J, Chen J. Efficient synthesis of eriodictyol from L-tyrosine in Escherichia coli. Appl Environ Microbiol. 2014;80:3072.

9. Trantas E, Panopoulos N, Ververidis F. Metabolic engineering of the complete pathway leading to heterologous biosynthesis of various flavonoids and stilbenoids in Saccharomyces cerevisiae. Metab Eng. 2009;11:355-66.

10. Leonard E, Yan Y, Koffas MA. Functional expression of a P450 flavonoid hydroxylase for the biosynthesis of plant-specific hydroxylated flavonols in Escherichia coli. Metab Eng. 2006;8:172-81.

11. Miyahisa I, Funa N, Ohnishi Y, Martens S, Moriguchi T, Horinouchi S. Combinatorial biosynthesis of flavones and flavonols in Escherichia coli. Appl Microbiol Biotechnol. 2006;71:53-8.

12. Janssen HJ. Fatty acid synthesis in Escherichia coli and its applications towards the production of fatty acid based biofuels. Biotechnol Biofuels. 2014;7:7.

13. Cronan JE, Thomas J. Bacterial fatty acid synthesis and its relationships with polyketide synthetic pathways. Complex Enzym Microbial Nat Prod Biosynth Part B Polyketides Aminocoumarins Carbohydr. 2009:459:395-433.

14. Leonard E, Lim KH, Saw PN, Koffas MA. Engineering central metabolic pathways for high-level flavonoid production in Escherichia coli. Appl Environ Microbiol. 2007;73:3877-86.

15. Liu W, Zhang B, Jiang R. Improving acetyl-CoA biosynthesis in Saccharomyces cerevisiae via the overexpression of pantothenate kinase and PDH bypass. Biotechnol Biofuels. 2017;10:41.

16. Owens DK, Crosby KC, Runac J, Howard BA, Winkel BSJ. Biochemical and genetic characterization of Arabidopsis flavanone $3 \beta$-hydroxylase. Plant Physiol Biochem Ppb. 2008;46:833-43.

17. Halbwirth H, Fischer TC, Schlangen K, Rademacher W, Schleifer K-J, Forkmann G, Stich K. Screening for inhibitors of 2-oxoglutarate-dependent dioxygenases: flavanone 3 $\beta$-hydroxylase and flavonol synthase. Plant Sci. 2006;171:194-205.

18. Falcone Ferreyra ML, Rius S, Emiliani J, Pourcel L, Feller A, Morohashi K, Casati P, Grotewold E. Cloning and characterization of a UV-B-inducible maize flavonol synthase. Plant J. 2010;62:77-91.

19. Pelletier MK, Murrell JR, Shirley BW. Characterization of flavonol synthase and leucoanthocyanidin dioxygenase genes in Arabidopsis. Further evidence for differential regulation of "early" and "late" genes. Plant Physiol. 1997:113:1437-45.

20. Lukačin R, Wellmann F, Britsch L, Martens S, Matern U. Flavonol synthase from Citrus unshiu is a bifunctional dioxygenase. Phytochemistry. 2003;62:287-92

21. Yang SM, Han SH, Kim BG, Ahn JH. Production of kaempferol 3-O-rhamnoside from glucose using engineered Escherichia coli. J Ind Microbiol Biotechnol. 2014:41:1311-8.

22. Prescott AG, Stamford NP, Wheeler G, Firmin JL. In vitro properties of a recombinant flavonol synthase from Arabidopsis thaliana. Phytochemistry. 2002;60:589-93.

23. Kim BG, Joe EJ, Ahn JH. Molecular characterization of flavonol synthase from poplar and its application to the synthesis of 3-O-methylkaempferol. Biotechnol Lett. 2010;32:579-84

24. Malla S, Pandey RP, Kim BG, Sohng JK. Regiospecific modifications of naringenin for astragalin production in Escherichia coli. Biotechnol Bioeng. 2013:110:2525-35.

25. Leonard E, Yan Y, Fowler ZL, Li Z, Lim CG, Lim KH, Koffas MAG. Strain improvement of recombinant Escherichia coli for efficient production of plant flavonoids. Mol Pharm. 2007;5:257-65.

26. Xu P, Ranganathan S, Fowler ZL, Maranas CD, Koffas MAG. Genomescale metabolic network modeling results in minimal interventions that cooperatively force carbon flux towards malonyl-CoA. Metab Eng. 2011;13:578-87.

27. Tehlivets O, Scheuringer K, Kohlwein SD. Fatty acid synthesis and elongation in yeast. Biochim Biophys Acta. 2007:1771:255-70.

28. Woods A, Munday MR, Scott J, Yang X, Carlson M, Carling D. Yeast SNF1 is functionally related to mammalian AMP-activated protein kinase and regulates acetyl-CoA carboxylase in vivo. J Biol Chem. 1994;269:19509-15.

29. Shi S, Chen Y, Siewers V, Nielsen J. Improving production of malonyl coenzyme A-derived metabolites by abolishing Snf1-dependent regulation of Acc1. MBio. 2014;5:e01130-14.

30. Shi S, Valle-Rodriguez JO, Khoomrung S, Siewers V, Nielsen J. Functional expression and characterization of five wax ester synthases in
Saccharomyces cerevisiae and their utility for biodiesel production. Biotechnol Biofuels. 2012;5:7.

31. Choi JW, Da Silva NA. Improving polyketide and fatty acid synthesis by engineering of the yeast acetyl-CoA carboxylase. J Biotechnol. 2014;187:56-9.

32. Kildegaard KR, Jensen NB, Schneider K, Czarnotta E, Ozdemir E, Klein T, Maury J, Ebert BE, Christensen HB, Chen Y, et al. Engineering and systemslevel analysis of Saccharomyces cerevisiae for production of 3-hydroxypropionic acid via malonyl-CoA reductase-dependent pathway. Microb Cell Fact. 2016;15:53.

33. Davis MS, Solbiati J, Cronan JE Jr. Overproduction of acetyl-CoA carboxylase activity increases the rate of fatty acid biosynthesis in Escherichia coli. J Biol Chem. 2000;275:28593-8.

34. Bhan N, Xu P, Khalidi O, Koffas MAG. Redirecting carbon flux into malonylCoA to improve resveratrol titers: proof of concept for genetic interventions predicted by OptForce computational framework. Chem Eng Sci. 2013;103:109-14.

35. Li X, Guo D, Cheng Y, Zhu F, Deng Z, Liu T. Overproduction of fatty acids in engineered Saccharomyces cerevisiae. Biotechnol Bioeng. 2014;111:1841-52.

36. Xu P, Li L, Zhang F, Stephanopoulos G, Koffas M. Improving fatty acids production by engineering dynamic pathway regulation and metabolic control. Proc Natl Acad Sci USA. 2014;111:11299-304.

37. Chen WN, Tan KY. Malonate uptake and metabolism in Saccharomyces cerevisiae. Appl Biochem Biotechnol. 2013;171:44-62.

38. Malla S, Koffas MA, Kazlauskas RJ, Kim BG. Production of 7-O-Methyl aromadendrin, a medicinally valuable flavonoid, in Escherichia coli. Appl Environ Microbiol. 2012;78:684-94.

39. Rodriguez A, Strucko T, Stahlhut SG, Kristensen M, Svenssen DK, Forster J, Nielsen J, Borodina I. Metabolic engineering of yeast for fermentative production of flavonoids. Bioresour Technol. 2017. doi:10.1016/j.biortech.

40. Tippmann S, Scalcinati G, Siewers V, Nielsen J. Production of farnesene and santalene by Saccharomyces cerevisiae using fed-batch cultivations with RQ-controlled feed. Biotechnol Bioeng. 2016;113:72-81.

41. Rodriguez A, Kildegaard KR, Li M, Borodina I, Nielsen J. Establishment of a yeast platform strain for production of $p$-coumaric acid through metabolic engineering of aromatic amino acid biosynthesis. Metab Eng. 2015;31:181-8.

42. Jiang NH, Zhang GH, Zhang JJ, Shu LP, Zhang W, Long GQ, Liu T, Meng ZG, Chen JW, Yang SC. Analysis of the transcriptome of Erigeron breviscapus uncovers putative scutellarin and chlorogenic acids biosynthetic genes and genetic markers. PLoS ONE. 2014;9:e100357.

43. Shubeita HE, Sambrook JF, Mccormick AM. Molecular-cloning and analysis of functional cdna and genomic clones encoding bovine cellular retinoic acid-binding protein. Proc Natl Acad Sci USA. 1987;84:5645-9.

44. Quan JY, Tian JD. Circular polymerase extension cloning for high-throughput cloning of complex and combinatorial DNA libraries. Nat Protoc. 2011:6:242-51

45. Li S, Ding W, Zhang X, Jiang H, Bi C. Development of a modularized twostep (M2S) chromosome integration technique for integration of multiple transcription units in Saccharomyces cerevisiae. Biotechnol Biofuels. 2016;9:232

46. Shiba Y, Paradise EM, Kirby J, Ro DK, Keasing JD. Engineering of the pyruvate dehydrogenase bypass in Saccharomyces cerevisiae for high-level production of isoprenoids. Metab Eng. 2007:9:160-8.

47. Gibson DG, Young L, Chuang RY, Venter JC, Rd HC, Smith HO. Enzymatic assembly of DNA molecules up to several hundred kilobases. Nat Methods. 2009;6:343-5.

48. Shao Z, Zhao H, Zhao H. DNA assembler, an in vivo genetic method for rapid construction of biochemical pathways. Nucleic Acids Res. 2009;37:e16.

49. Paddon CJ, Westfall PJ, Pitera DJ, Benjamin K, Fisher K, McPhee D, Leavel MD, Tai A, Main A, Eng D, et al. High-level semi-synthetic production of the potent antimalarial artemisinin. Nature. 2013:496:528-32.

50. Gietz RD, Akio S. New yeast-Escherichia coli shuttle vectors constructed with in vitro mutagenized yeast genes lacking six-base pair restriction sites. Gene. 1989;74:527-34.

51. Thomas BJ, Rothstein R. Elevated recombination rates in transcriptionally active DNA. Cell. 1989;56:619. 